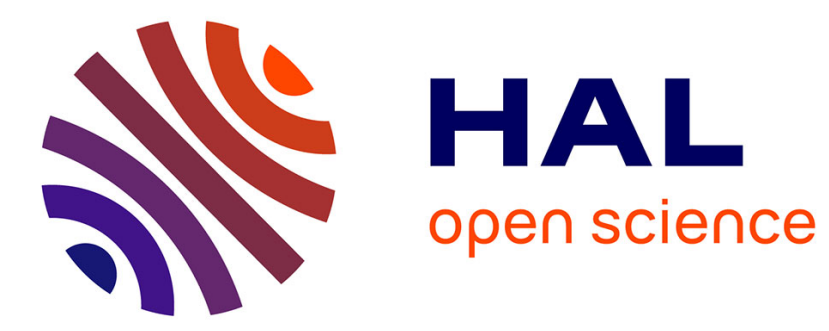

\title{
Fuzzy Neural Network with Support Vector-based Learning for Classification and Regression
}

\author{
Mohammad Mehdi Ebadzadeh, Ghazaleh Khodabandelou
}

\section{To cite this version:}

Mohammad Mehdi Ebadzadeh, Ghazaleh Khodabandelou. Fuzzy Neural Network with Support Vector-based Learning for Classification and Regression. Soft Computing, 2019, 23 (23), pp.1215312168. 10.1007/s00500-019-04116-x . hal-02962536

\section{HAL Id: hal-02962536 https://hal.sorbonne-universite.fr/hal-02962536}

Submitted on 9 Oct 2020

HAL is a multi-disciplinary open access archive for the deposit and dissemination of scientific research documents, whether they are published or not. The documents may come from teaching and research institutions in France or abroad, or from public or private research centers.
L'archive ouverte pluridisciplinaire $\mathbf{H A L}$, est destinée au dépôt et à la diffusion de documents scientifiques de niveau recherche, publiés ou non, émanant des établissements d'enseignement et de recherche français ou étrangers, des laboratoires publics ou privés. 


\title{
Fuzzy Neural Network with Support Vector-based Learning for Classification and Regression
}

\author{
Ghazaleh Khodabandelou ${ }^{\mathrm{a}, *}$, Mohammad Mehdi Ebadzadeh ${ }^{1, \mathrm{~b}}$ \\ ${ }^{a}$ Theoretical physics of condensed matter (LPTMC), Sorbonne Universités, UPMC, 4 place Jussieu, 75005, Paris, \\ France \\ ${ }^{b}$ Computer Engineering Department, Amirkabir University of Technology, Hafez street, Tehran, Iran
}

\begin{abstract}
Fuzzy Neural Network (FNN) and Support Vector Machine (SVM) are two prominent and powerful learning models broadly used for classification and regression. FNN has a significant local representation and human reasoning advantage. However, the drawback of such a network is that the focal point of the learning algorithms (e.g., backpropagation) is minimizing empirical risk. In contrary to FNN, SVM emphasizes simultaneously on minimizing empirical and expected risks, which theoretically leads to an excellent generalization performance power. Nevertheless, this depends on determining an adequate kernel function which is consistent with the data properties. Some approaches have been proposed to take advantage of one to deal with disadvantage of the other by combining SVM kernel and FFN. In this paper, we show that a Takagi-Sugeno-Kang (TSK)-type-based fuzzy neural network is, in fact, equivalent to an SVM with an adaptive kernel based on fuzzy rules generated in this FNN. Consequently, it is possible to learn the last layer of the FNN using the concepts of SVM and thus taking the advantage of SVM in generalization. In fact, on the one side, the proposed method is an SVM with an adaptive kernel based on fuzzy rules and on the other side, it is a TSK-FNN with SVM-based learning. Therefore, the FNN with SVM-based learning has the benefit of SVM which is minimizing both learning and testing errors. Besides, such FNN does not have the disadvantages of the SVM which is finding an appropriate kernel and training the kernel parameters. As a matter of fact, the defined kernel in FNN is an adaptive kernel based on data characteristics, which is derived from the fuzzy rules generated by the FNN itself. As a result, it is expected that the results of the proposed method for classification and regression outperform the results of SVM with the conventional kernels and fuzzy neural network trained in the conventional way. This claim is confirmed with the results obtained in this paper.
\end{abstract}

Keywords: Fuzzy neural network (FNN), Support vector machine (SVM), Adaptive fuzzy kernel, Classification, Regression

\section{Introduction}

The two most salient techniques for pattern classification and regression are the Fuzzy neural network (FNN) and support vector machine (SVM). SVM is a kernel-based machine learning method which is based on the statistical learning theory [1. The focus of SVM, in contrary to FNN, is 5 simultaneously on minimizing empirical and expected risks. This theoretically leads to an excellent generalization performance strength. Nevertheless, this highly depends on determining an adequate kernel function that is consistent with the data properties [2]. Determining manually an appropriate

\footnotetext{
${ }^{*}$ Corresponding author

Email address: ghazaleh@lptmc.jussieu.fr, ghazaleh.khodabandeh@gmail.com (Ghazaleh Khodabandelou)
} 
kernel function or adjusting its parameters is an exhaustive task since it requires specific knowledge of the field. This issue has been addressed in several studies by automatically identifying the proper kernel using prior knowledge [3, 4, 15, 6, 7].

A fuzzy system can estimate any continuous real function specified on a bounded domain as shown in [8]. Using a collection of fuzzy rules the fuzzy system covers the function graph in input-output data. Integration of fuzzy system to neural networks has given birth to hybrid fuzzy neural networks (FNNs) [9]. These networks are considered as a particular type of neural networks due to their similarity to neurons weights represent rules in the fuzzy system. In this regards, the adaptive-network-based fuzzy inference systems (ANFIS) has been proposed in [10] where the system is formulated based on both specified input-output space and humans' cognition. This system has yielded significant performance and since has been extensively used in modeling and handling nonlinear systems [11. ANFIS uses 20 Takagi-Sugeno-Kang fuzzy system [12] with combining gradient decent and least square method in the learning procedure [13. Nevertheless, ANFIS is affected by the curse of dimensionality when faced with the large number of input dimension (i.e., the number of fuzzy rules). Therefore, it is necessary to integrate into the learning algorithm a reliable method to reduce the number of fuzzy rules. FNNs have some advantages such as human-like reasoning and remarkable local representation [14, 15]. However,

\section{the drawback of such networks is that their learning algorithms (i.e., back-propagation) only focus} on minimizing empirical risk [16, 17]. Furthermore, a major challenge of existing FNN methods is dealing with a large number of fuzzy rules. Therefore, several approaches have been proposed in the literature for optimizing the number of rules in hybrid FNN such as dividing the input space by some unsupervised algorithms [18, 19] or by clustering algorithms [20, 21]. Some methods proposed to consider both input and output data to determine fuzzy rules [22, 23]. In [24, 25] the cluster number has been manually specified, which is not guaranteed to return an optimal solution. All these methods are human-in-the-loop systems which require prior establishment of fuzzy rules numbers by an expert. Consequently, to overcome this issue dynamic adaptive methods have been proposed in several studies [24, 16, 14, 26, 27]. Some efforts have been made to surmount the challenges in SVM and FNN by leveraging the properties of both techniques (i.e., by developing a theoretical foundation of FNN using the SVM learning theory) [28, 29, 30, 31, 32. Despite the fact that all these methods use an elimination procedure to reduce the number of fuzzy rules, they still require a high number of fuzzy rules to achieve high learning performance. Besides, it is crucial that these fuzzy rules be provided automatically by an adaptive fuzzy system.

In order to address the aforementioned challenges, we leverage the underlying relation between SVM kernel and FNN. We propose a new combinatorial method, so-called Fuzzy Neural Network with Support Vector-based learning (FNN-SVBL), to take advantage of this underlying relation. In this paper, we show that a TSK-type fuzzy neural network is, in fact, equivalent to an SVM with an adaptive kernel based on fuzzy rules generated by TSK-FNN. Therefore, it is possible to learn 45 the last layer of the TSK-FNN using the concepts of SVM and thus obtain the benefits of SVM in generalization. In fact, on the one side, the proposed method is an SVM with an adaptive kernel based on fuzzy rules and on the other side, it is a TSK-FNN with SVM-based learning in the last layer. Therefore, the FNN with SVM-based learning has the advantages of SVM, which minimizes both learning and testing errors. Besides, such FNN does not have the disadvantages of the SVM which is finding an appropriate kernel and training the kernel parameters. As a result, the defined kernel in FNN is an adaptive kernel based on data characteristics, which is derived from the fuzzy rules generated by the FNN itself. We use a Gaussian Mixture Model (GMM) to generate the fuzzy rules of FNN-SVBL. The number of fuzzy rules for each dataset is determined as in [33]. For evaluating the classification performance of FNN-SVBL we use some well-known benchmark datasets. These datasets 55 are accessible at the UCI repository of machine learning databases [34] and the Statlog collection [35. Also, we use two simulated datasets for the regression performance assessment. Finally, we assess the 
sensitivity of the proposed method to the number of fuzzy rules. As the experimental results delineate the classification and regression performances of FNN-SVBL are competitive or even superior to stateof-the-art methods while the fuzzy rules number is substantially lower than those methods.

The remaining sections of the paper are organized as follows. In Section 2, we discuss briefly some related previous works. Section 3 and 4 briefly reviews the SVM learning theory and the GMM algorithm, respectively. In Section 5, we describe the fuzzy neural network. In Section 6 we explain the learning method based on support vector for the last layer of the FNN-SVBL. In Section 7, we present and discuss in detail, some experimental results and comparisons. Finally, in Section 8, we conclude the paper.

\section{Related works}

In this section, we review the state-of-the-art of existing work focusing on combination of fuzzy theory techniques and kernel learning.

\subsection{Positive-definite kernel functions on fuzzy sets}

Some approaches have been dealing with popular machine learning problems such as classification [28, 32], clustering [36, 37], feature extraction [38, discriminant analysis [39] by combining fuzzy techniques with positive definite kernels. Incorporating non-vectorial fuzzy data into a normed vectorial space such as kernel Hilbert space (RKHS) has given birth to some innovating methods [40, 41, which use a positive definite kernel function specified on fuzzy sets. The kernel proposed in [42 measures the resemblance between fuzzy sets in RKHS. It has been demonstrated in this approach that such a kernel is a special case of the convolution kernel. An improvement of [43] has been proposed in [44] to deal with the sensitivity problem of clustering in the presence of outliers.

\subsection{SVM kernel learning}

Several methods of SVM kernel learning have been proposed in the literature. These methods aim The approach proposed in 6 has chosen a classic kernel in order to specify the kernel parameters by optimizing its quality function. Some methods have been defined the quality function as class separability [45, 46], Bayesian probabilities [47, 48], generalization error [3], alignment-based techniques [49, 4] or cross-validation error [50]. Certain approaches have been combined the classic kernels 85 [51, 7, 52. The combination coefficients can be estimated through convex optimization. However, this approach is time-consuming and resource intensive. An inductive approach, known as multiple kernel learning, consists in learning a combined kernel from a restricted classic kernels set [53, 54, 5]. Nevertheless, there are a limited learnable kernels class. In [32] a customized similarity measurement function has been used to estimate the kernel matrix inputs. Some methods have been proposed to set up the SVM kernel function [3, 50, 47, 32].

\subsection{Combination of fuzzy systems and SVM}

A widely known method, called FSVMs, takes advantage of both SVM and fuzzy systems by redefining the SVM optimization problem using fuzzy theory. In this method, a fuzzy membership value is applied to each input point such that these latter participate differently to the learning of decision surface [55]. Similarly, some methods have been proposed to include the membership value of each input point in the penalty term [56, 57]. The proposed method in [56] attempts to improve FSVMs for handling the class imbalance in the presence of outliers and noise. Although using these methods contributes to reduce the class imbalance effect, the principal issue is finding a way to assess an adequate fuzzy membership [58]. An interval type-2 fuzzy support vector machine has been proposed in [59] to classify patients to three classes. In [60] a hybrid classifier has been proposed 
which is composed of unsupervised fuzzy clustering c-mean and supervised support vector machine. In a multiclass context, an approach has been proposed in [61 to determine classes in "unclassifiable regions". This approach consists in specifying a fuzzy membership function for each class found on the SVM separating hyperplane. In [62] the authors have studied a new clustering algorithm to achieve a more solid fuzzy membership for fuzzy SVM. They have proposed to ameliorate the partition index maximization by obtaining appropriate data centroid. The fuzzy SVM has been used to influence the classification decision function.

\subsection{SVM-based fuzzy neural networks}

Several approaches, including the proposed method in this paper, focus on expanding a theoretical foundation for FNN based on the SVM learning theory. In [32] a support vector-based fuzzy neural network has been proposed for patterns classification. First, the fuzzy rules and membership functions were defined using clustering rules. Second, the parameters of FNN were estimated by the SVM. Finally, the fuzzy rules were chosen by the proposed fuzzy rule method by omitting irrelevant fuzzy rules. An SVM has been used with a Gaussian kernel on a task training set in [28, 29. In these 115 approaches, the extracted support vectors were used to generate fuzzy rules of FNN. The proposed approach in [31] has tackled the problem of minimizing the training and testing errors simultaneously in a pattern classification context. To this end, a support-vector-based fuzzy neural network classification was proposed using jointly the classification strength of SVM and the efficient human-like reasoning of FNN. However, the main drawback of these approaches [31, 29, 28] is when the fuzzy rules number becomes equivalent to the support vectors number, which is generally very large. In all aforementioned methods, the consequent of fuzzy rules is of the fuzzy singleton type. A Takagi-Sugeno fuzzy system for a classification objective has been proposed in [63] which uses a linear SVM to tune the PCA parameters. A fuzzy system was proposed in [30] which is generated by the crossbreeding of fuzzy clustering and SVM. Here, the assumption is that a fuzzy system with Takagi-Sugeno outperforms a fuzzy system with the fuzzy singletons, as shown in [64, 65]. In [66] the authors have studied the relationship among non singleton fuzzy input, TSK fuzzy systems, and positive definite kernels.

\section{Support vector machine}

The learning theory of SVM has been developed in [1]. In this section, we describe the learning process of SVM in a binary classification issue. Let $S=\left\{\left(X_{1}, y_{1}\right),\left(X_{2}, y_{2}\right), \cdots,\left(X_{n}, y_{n}\right)\right\}$ be a set of training data, where $X_{i} \in \mathcal{R}^{d}$. Each input $X_{i}$ is a d-dimensional real vector and $y_{i} \in\{-1,+1\}$ stands for the input labels. SVM is a classifier specified by a separating hyperplane which tends to maximize the margin width between two classes. For linearly separable data the hyperplane is defined as:

$$
y_{i}\left(W^{T} X_{i}+b\right) \geq 1-\varepsilon_{i}, \quad i=\{1, \cdots, n\}, \quad \varepsilon_{i} \geq 0
$$

where $W \in \mathcal{R}^{d}, b \in \mathcal{R}$ and $\varepsilon_{i} \geq 0$. The slack variable is presented by $\varepsilon_{i}$ which is the smallest nonnegative number satisfying Eq.(1). In cases where values of $\varepsilon_{i}>1$ the data are misclassified. Therefore, to discover the separating hyperplane having the maximum margin, the constrained optimization problem with a differentiable objective function can be solved as follows:

$$
\begin{array}{r}
\arg \min \frac{1}{2}\|W\|^{2}+C \sum_{i=1}^{n} \varepsilon_{i} \\
\text { subject to } \quad y_{i}\left(W^{T} X_{i}+b\right) \geq 1-\varepsilon_{i}, \quad i=\{1, \cdots, n\}
\end{array}
$$


where $\varepsilon_{i}$ stands for a slack variable for the $i$ th sample, $C$ is a cost parameter and $\sum_{i=1}^{n} \varepsilon_{i}$ stands for an upper bound of the expected training error (the primal problem). By constructing a Lagrangian representation for the above quadratic optimization problem, one obtains the following dual problem:

$$
\begin{array}{r}
\arg \max \left(\sum_{i=1}^{n} \alpha_{i}-\frac{1}{2} \sum_{i=1}^{n} \sum_{j=1}^{n} \alpha_{i} \alpha_{j} y_{i} y_{j} X_{i}^{T} X_{j}\right) \\
\text { subject to } \quad \sum_{i=1}^{n} \alpha_{i} y_{i}=0, \quad \alpha_{i} \geq 0, \quad i=\{1, \cdots, n\}
\end{array}
$$

where $\alpha_{i}$ are Lagrange multipliers and the dual maximization problem is a quadratic function of the $\alpha_{i}$ subject to linear constraints. One obtains $\alpha_{i}$ by solving Eq.(3) so that the final hyperplane decision function is achieved as follow:

$$
f(X)=\operatorname{sign}\left\{W^{T} X+b\right\}=\operatorname{sign}\left\{\sum_{X_{i} \in S V} \alpha_{i} y_{i} X_{i}^{T} X+b\right\}
$$

where $b$ is the offset and $S V$ are the support vectors, with the nonzero $\alpha_{i}$ values in the training samples set. The derivation process is explained in detail in [6]. In order to maximize the separability among classes mainly for non-linearly separable data, one can use a mapping function $\phi(X)$ to transform the data into a higher dimensional feature space. To do so, $X_{i}$ in Eq.(1), (2), (3) and (4) can be transformed into $\phi\left(X_{i}\right)$ and $X$ in Eq. (4) into $\phi(X)$. The SVM allows for defining implicitly the mapping function by applying a kernel function such that $K\left(X_{i}, X_{j}\right)=\phi\left(X_{i}\right)^{T} \phi\left(X_{j}\right)$. Therefore, the dual optimization problem in Eq.(3) can be transformed into its kernel form. The final decision function is defined as follows:

$$
f(X)=\operatorname{sign}\left\{\sum_{X_{i} \in S V} \alpha_{i} y_{i} k\left(X_{i}, X\right)+b\right\}
$$

where $b$ can be established by the Karush-Kuhn-Tucker (KKT) complementary slackness conditions [1].

\section{Gaussian mixture model clustering algorithm}

Clustering algorithms can be widely categorized into two classes: model-based and non-modelbased algorithms. Model-based clustering makes an assumption that an underlying model has generated the data and attempts to discover the original model from the data. This kind of clustering is based on a limited mixture of distributions in which each mixture component is related to a different cluster 67]. A commonly used algorithm in model-based clustering context is the ExpectationMaximization (EM) algorithm which allows estimating the finite mixture models [68]. The Bayesian information criterion is widely used to determine the number of components in a Gaussian Mixture. Other approaches are cross-validation or heuristic-based greedy [69]. In non-model-based clustering algorithms, which are based on the distance and similarity between clusters, the assumption is the data do not follow a particular model [70, 27]. In this paper, we use the Gaussian mixture model algorithm (GMM) that is a probabilistic model assuming all the data points are generated from a mixture of a limited number of Gaussian distributions with unknown parameters. A GMM is usually used to 
estimate the distribution of random variables as a sum of several Gaussians. An iterative procedure optimizes the Gaussians parameters (e.g., variance, average, and amplitude) via a maximum likelihood criterion. This procedure is done using the EM algorithm. It should be pointed out that for the sake of comparison, we use also Fuzzy c-means (FCM) and Hierarchical Cluster Analysis (HCA) for both classification and regression. The results are reported in section 7.5 .

\section{Fuzzy neural network}

A fuzzy system consists of a collection of fuzzy rules utilizing human knowledge processing ability. Fuzzy neural networks (FNNs) exploit both the fuzzy system and neural networks abilities. On the one hand, FNNs learning algorithm is inspired by the ability of neural network theory in learning from processes, on the other hand, FNNs use fuzzy systems reasoning in handling unknown information. Fuzzy rules are composed of different preconditions assessing the association of the input variables to fuzzy sets and one consequence (i.e., fuzzy singleton, fuzzy sets or TSK-type consequence). As shown in 65, 64 facing sophisticated problems a FNN acquiescent with TSK-type consequence usually outperforms fuzzy singleton or fuzzy set consequence. Each rule in a TSK-type fuzzy system is defined as follows:

$$
\begin{aligned}
& \text { Rule } c: \text { IF } x_{1} \text { is } A_{c 1} \text { AND } \cdots \text { AND } x_{d} \text { is } A_{c d} \\
& \qquad \text { THEN } y=\beta_{c 0}+\sum_{i}^{d} \beta_{c i} x_{i}, \quad c=\left\{1, \cdots, n_{c}\right\}
\end{aligned}
$$

where $A_{c i}$ represents the fuzzy set of the input variables $x_{i}, \beta_{c i}$ is a real number, $n_{c}$ is the number of fuzzy rules and $d$ is the number of dimensions. As depicted in Fig 1, the network structure of the proposed model (FNN-SVBL) is a FNN with fuzzy rules consequences of TSK-type. FNN-SVBL consists of five layers including the data input, membership function, rule, consequent and output layers, respectively. The first step is to use GMM clustering algorithm to construct the FNN fuzzy rules by dividing input space into overlapping clusters. In fact, the generated clusters correspond to rules in the rule-base. This step is necessary to tune the parameters of FNN-SVBL by constructing the SVM kernel. The clustering of input space has to be applied on the basis that the points in a cluster have the same values or belong to the same class of the output variable. To this end, first, the training set is partitioned into two subsets using class information such that each subset comprises the same class samples. Second, GMM is applied to each subset to obtain two categories of clusters. In the training phase, the class information is explicitly considered to construct fuzzy rules. In the testing phase, the input data influence directly fuzzy rules with no need for such information. The parameters of the Gaussian function for a cluster $c$ in the input space are represented by $\mu_{c}$ and $\Sigma_{c}$, which stand for mean and diagonal covariance matrix of samples, respectively. Thereby, the Gaussian function is represented as follows:

$$
g_{c}(X)=\exp \left\{-\gamma\left(X-\mu_{c}\right)^{T} \Sigma_{c}^{-1}\left(X-\mu_{c}\right)\right\}
$$

where the parameter $\gamma$ stands for the inverse of the radius of influence of samples selected by the model as support vectors. The preconditions of the fuzzy rules are determined using the clusters generated in the previous step. Since GMM generates orthogonal clusters, the membership functions can be achieved by projecting them onto the input variables in the cluster space.

Let $I^{(L)}$ and $O^{(L)}$ be the input and output variables of a node in layer $L$ of the FNN-SVBL network, respectively. In what follows, we describe the basic functions and the signal propagation in each layer of the network: 


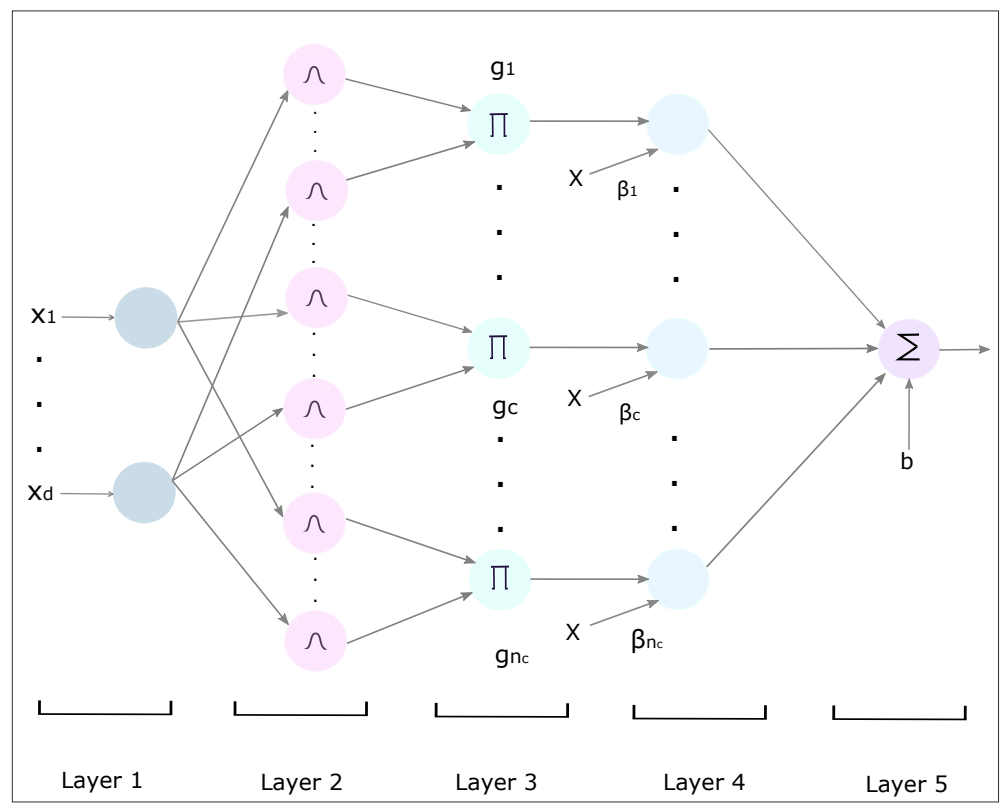

Figure 1: The network structure of the proposed model (FNN-SVBL).

Layer 1 - Input layer. This layer is the input layer and each node represents an input variable. In this layer, the input values are directly forwarded towards the next layer. The output of this layer is defined as:

$$
O_{i}^{(1)}=I_{i}^{(1)}=x_{i}
$$

where $x_{i}$ is the $i$ th input variables of the network.

Layer 2 - Membership function layer. In this layer, nodes represent membership functions corresponding to a fuzzy set of the input variables. Indeed, the membership functions evaluate the membership degree of input values to that fuzzy set. The membership value of each node is calculated as follows:

$$
O_{c_{i}}^{(2)}=\exp \left\{\frac{-\left(x_{i}-\mu_{c_{i}}\right)^{2}}{\sigma_{c_{i}}^{2}}\right\}=M_{c_{i}}\left(x_{i}\right)
$$

where $O_{c_{i}}^{(2)}$ stands for the output of the $i$ th membership function of the $c$ th cluster (rule).

Layer 3 - Rule layer. In this layer, nodes represent fuzzy logic rules and carry out fuzzy preconditioning of the rules. In fact, the output of this layer represents the firing force of a fuzzy rule. The AND operation is applied to all nodes of this layer as follows:

$$
O_{c}^{(3)}=\prod_{i=1}^{d} I_{c_{i}}^{(3)}=\prod_{i=1}^{d} O_{c_{i}}^{(2)}=\prod_{i=1}^{d} M_{c_{i}}\left(x_{i}\right),
$$

When $X$ is the network input vector, $g_{c}(X)$ in Eq.77 is equivalent to $O_{c}^{(3)}$. Thus, the Eq. 10 can 
be defined as follows:

$$
\begin{aligned}
O_{c}^{(3)} & =\prod_{i=1}^{d} \exp \left\{-\frac{\left(x_{i}-\mu_{c_{i}}\right)^{2}}{\sigma_{c_{i}}^{2}}\right\} \\
& =\exp \left\{-\sum_{i=1}^{d} \frac{\left(x_{i}-\mu_{c_{i}}\right)^{2}}{\sigma_{c_{i}}^{2}}\right\} \\
& =\exp \left\{-\left(X-\mu_{c}\right)^{T} \Sigma_{c}^{-1}\left(X-\mu_{c}\right)\right\}=g_{c}(X),
\end{aligned}
$$

where $\Sigma_{c}$ is a diagonal matrix such that each diagonal element of $\Sigma_{c_{i i}}=\sigma_{c_{i}}^{(2)}$.

Layer 4 -Consequent layer. In this layer, the TSK-type consequence of the fuzzy rules are generated by the nodes. A linear combination of the input vector $\beta_{c}^{T} X$ is applied to $c$ th rule (node), where $\beta_{c}$ represents the network parameter to be adjusted by SVM. The output value of the $c$ th node is as follows:

$$
O_{c}^{(4)}=\left(\beta_{c 0}+\beta_{c}^{T} X\right) \times \exp \left\{-\left(X-\mu_{c}\right)^{T} \Sigma_{c}^{-1}\left(X-\mu_{c}\right)\right\} .
$$

Layer 5 - Output layer. In this layer, the global output is calculated as the weighted sum of all the inputs with a bias term. The output of this layer is as follows:

$$
\begin{aligned}
O^{(5)} & =\sum_{c=1}^{n_{c}}\left(\beta_{c 0}+\beta_{c}^{T} X\right) \times \exp \left\{-\left(X-\mu_{c}\right)^{T} \Sigma_{c}^{-1}\left(X-\mu_{c}\right)\right\}+b \\
& =\sum_{c=1}^{n_{c}}\left(\beta_{c 0}+\beta_{c}^{T} X\right) \times g_{c}(X)+b
\end{aligned}
$$

Consequently, the decision function of FNN-FVBL network can be written as follows:

$$
f(X)=\operatorname{sign}\left\{\sum_{c=1}^{n_{c}}\left(\beta_{c 0}+\beta_{c}^{T} X\right) \times g_{c}(X)+b\right\}
$$

In the next section, it will be shown that the Eq.(14) can be equivalent to the Eq.(5), or, in other words, the parameters of $\beta$ in the FNN can be written in terms of the parameters of $\alpha$ in the SVM. Therefore, instead of learning the values of $\beta$ in the FNN, one can learn the values of $\alpha$ in the SVM, and then, based on their underlying relationship, the values of $\beta$ can be obtained from the values of $\alpha$.

\section{Proposed method}

In this section, we show that the Eq.(14) can be equivalent to the Eq.(5), or in other terms, a FNN equivalent to an SVM with an adaptive fuzzy rule-based kernel. If we consider the Eq. (14) obtained in the previous section, which is the output equation of the FNN, then we have:

$$
f(X)=\sum_{c=1}^{n_{c}}\left(\beta_{c 0}+\beta_{c}^{T} X\right) g_{c}(X)+b
$$


with considering :

$$
\begin{aligned}
\beta_{c 0} & =\sum_{i=1}^{S V} \alpha_{i} y_{i} g_{c}\left(X_{i}\right), \\
\beta_{c} & =\sum_{i=1}^{S V} \alpha_{i} y_{i} g_{c}\left(X_{i}\right) X_{i}
\end{aligned}
$$

by replacing Eq.(16) in Eq.(15), we obtain:

$$
\begin{aligned}
f(X) & =\sum_{c=1}^{n_{c}} \sum_{i=1}^{S V} \alpha_{i} y_{i} g_{c}\left(X_{i}\right) g_{c}(X)\left(1+X_{i}^{T} X\right)+b \\
& =\sum_{i=1}^{S V} \alpha_{i} y_{i}\left(1+X_{i}^{T} X\right) \sum_{c=1}^{n_{c}} g_{c}\left(X_{i}\right) g_{c}(X)
\end{aligned}
$$

with comparing Eq.(5) and Eq.(17) then:

$$
K\left(X_{i}, X\right)=\left(1+X_{i}^{T} X\right) \sum_{c=1}^{n_{c}} g_{c}\left(X_{i}\right) g_{c}(X)
$$

where $K: \mathbb{R}^{d} \times \mathbb{R}^{d} \rightarrow \mathbb{R}_{0}^{+}$, functions $g_{c}\left(X_{i}\right)$ and $g_{c}(X)$ determine the membership degree of a sample to cluster $c$ and $n_{c}$ represents the number of clusters.

The kernel $K\left(X_{i}, X\right)$ is adaptive since the number of Gaussian functions in $g_{c}\left(X_{i}\right)$ and $g_{c}(X)$ along with their parameters are automatically determined according to data distribution of the problem. Indeed, in the clustering phase, the data distribution is approximated by a set of Gaussian functions.

\section{Experimental results}

In this section, we first explain the benchmark datasets used to evaluate the method robustness. In order to evaluate the classification and regression performance, we then use conventional metrics. Last, we verify parameter sensitivity of FNN-SVBL on some datasets.

\subsection{Classification datasets}

In order to perform experiments for classification problem, we use six widely-used benchmark datasets accessible at the Statlog collection [35] and the UCI repository of machine learning databases 34. Hereafter, we describe briefly the chosen datasets. We select DNA, Vehicle and Satimage datasets from Statlog collection. The DNA dataset is a collection of 3186 feature vectors with 180 sequential DNA nucleotide positions as attributes. The dataset is split into 2000 samples for training and 1186 samples for testing. The data are classified into three classes. The DNA examples are taken from Genbank 64.1. The Vehicle dataset consists of 846 samples distributed among four classes. Each sample is represented by 18 input attributes. The Satimage dataset consists of the Landsat multispectral values of pixels in $3 \times 3$ neighborhoods in a satellite image. It contains 4435 instances for the training set and 2000 instances for the test set. The dataset is classified into six classes. Each sample is composed of 36 input numerical attributes in the range between 0 to 255 .

The Iris, Glass, and Wine datasets are selected from the UCI Repository. The Iris is the well-known dataset for pattern recognition problems. It contains 150 equally distributed samples each of them is characterized by four attributes information: septal length, septal width, petal length and petal width 


\subsubsection{Nonlinear sinc function}

The non-linear sinc function is defined as follows:

$$
f(x, y)=\mathcal{Z}=\frac{\sin (x) \sin (y)}{x y}, \quad x \in[-10, \cdots, 10], \quad y \in[-10, \cdots, 10]
$$

Using the non-linear sinc function a set of 250 samples is uniformly generated. This data are divided into 125 samples for each of training and test sets. Figure 2a depicts the surface of the training samples.

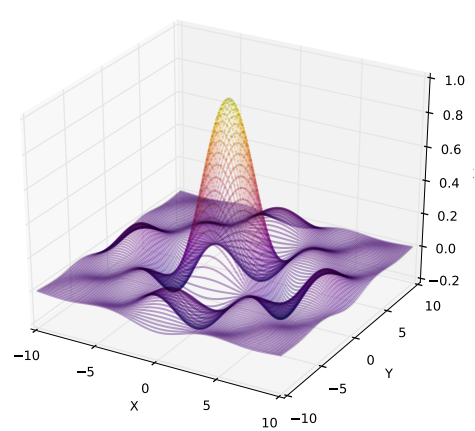

(a) Training samples

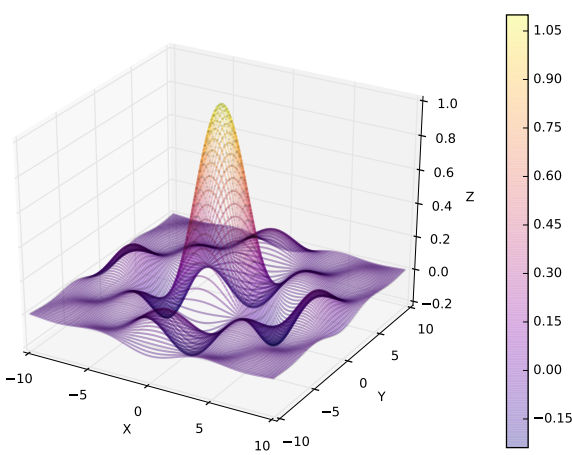

(b) Simulation

Figure 2: Nonlinear sinc surface data.

\subsubsection{Nonlinear dynamic system identification}

The Nonlinear dynamic system is defined as follows:

$$
y(t+1)=\frac{y(t) y(t-1)[y(t)+2.5]}{1+y^{2}(t)+y^{2}(t-1)}+u(t) \quad t \in[1,200], y(0)=0, y(1)=0
$$

where $u(t)=\sin \left(\frac{2 \pi t}{25}\right)$. The Eq. 20 can be described in a form of three-input one output function:

$$
\hat{y}(t+1)=f(y(t), y(t-1), u(t))
$$

A set of 200 data are selected as a training set and 200 data as a testing set. Figure 3a shows the surface from training points. 


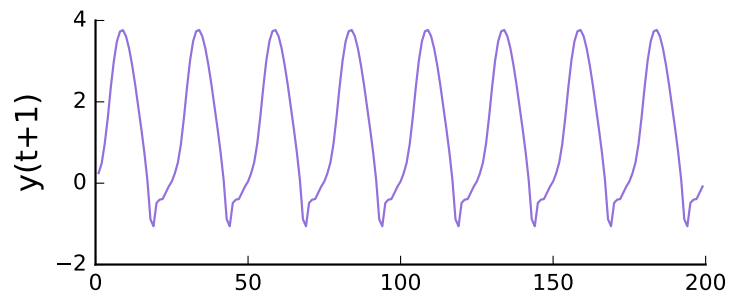

(a) Training samples

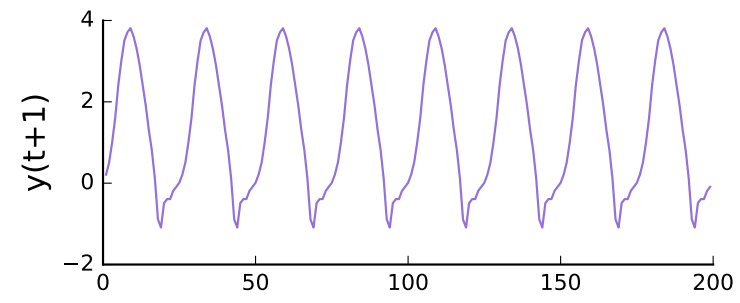

(b) Simulation

Figure 3: Nonlinear dynamic system data.

Table 1: The optimum cost parameter $C$ and kernel parameter $\gamma$ for the datasets used for classification.

\begin{tabular}{|c|c|c|c|c|c|c|c|c|}
\hline \multirow[b]{2}{*}{ Dataset } & \multicolumn{2}{|c|}{ FNN-SVBL } & \multicolumn{2}{|c|}{ SVFC-AK 33] } & \multicolumn{2}{|c|}{ RBF-SVM 26$]$} & \multirow{2}{*}{$\frac{\text { SVFNN }[32]}{C}$} & \multirow{2}{*}{$\frac{\text { SOTFN-SV }[64]}{C}$} \\
\hline & $C$ & $\gamma$ & $C$ & $\gamma$ & $C$ & $\gamma$ & & \\
\hline Iris & $2^{-1}$ & $2^{-1}$ & $2^{12}$ & $2^{-7}$ & $2^{12}$ & $2^{-9}$ & $2^{12}$ & $2^{-1}$ \\
\hline Vehicle & $2^{-3}$ & $2^{-1}$ & $2^{6}$ & $2^{-4}$ & $2^{9}$ & $2^{-3}$ & $2^{11}$ & $2^{-1}$ \\
\hline DNA & $2^{-11}$ & $2^{-1}$ & $2^{-3}$ & $2^{-8}$ & $2^{3}$ & $2^{-6}$ & $2^{4}$ & $n r^{*}$ \\
\hline Satimage & $2^{-9}$ & $2^{-1}$ & $2^{-1}$ & $2^{-7}$ & $2^{4}$ & $2^{0}$ & $2^{6}$ & $n r$ \\
\hline Glass & $2^{-1}$ & $2^{-1}$ & $2^{11}$ & $2^{-4}$ & $2^{12}$ & $2^{-4}$ & $n r$ & $n r$ \\
\hline Wine & $2^{-6}$ & $2^{-1}$ & $2^{3}$ & $2^{-4}$ & $2^{7}$ & $2^{-10}$ & $n r$ & $n r$ \\
\hline
\end{tabular}

${ }^{*} n r:$ is not reported.

\subsection{Performance comparison}

In this section, we compare the performance of FNN-SVBL with some of the state-of-the-art methods in terms of number of fuzzy rules or support vectors as well as the accuracy and Root Means Square Error (RMSE), which is defined as $\sqrt{\frac{\sum_{i=1}^{N}\left(x_{i}-\hat{\left.x_{i}\right)^{2}}\right.}{N}}$.

For both datasets used in classification and regression problems, we carry out the following steps: first, we normalized the datasets using z-score normalization formula: $\frac{x_{i}-\mu}{\sigma}$, where $x_{i}$ is the datasets samples, $\mu$ and $\sigma$ stand for mean and standard deviation of samples, respectively. Note that datasets with binary attribute (e.g., DNA) are not normalized. This scaling adjusts the values measured on different scales to a common one. Second, to evaluate the quality of our trained model, we use 10fold cross-validation. To this end, we estimate generalized accuracy and RMSE for classification and regression problems, respectively, by varying the cost parameters $C=\left[2^{-21}, \cdots, 2^{21}\right]$. The kernel parameter $\gamma$ is set to $2^{-1}$ since there is no changes in its other values. This procedure is applied over the training sets with tenfold cross-validation for each value of $C$. Then, to assess the quality of the model over unseen data, we apply the trained model with $C$ that reaches the optimal average cross-validation rate over the test sets. These parameters are reported in Table 1 for all datasets.

For datasets that do not explicitly contain test sets (e.g., Iris, Vehicle, Glass, and Wine), we split the whole dataset into halves as training and test sets. The proposed method (FNN-SVBL) is then applied over the training sets of these datasets using the number of fuzzy rules inspired from [33].

We report the optimal kernel and cost parameters for FNN-SVBL and the other methods in Table 1. The number of fuzzy rules/support vectors are reported in Table 2. The smallest and the largest numbers of fuzzy rules belong to Iris and Satimage, respectively. In general, FNN-SVBL and SVFCAK sketch the lowest fuzzy rules compared with the other methods.

The proposed method is then compared to some prominent related work in classification problem such as SVFC-AK [33], RBF kernel-based SVM [26], support vector-based fuzzy neural network 
Table 2: Number of support vectors (SV) or/and fuzzy rules (FR) for FNN-SVBL and the related work methods.

\begin{tabular}{cccrccc}
\hline Dataset & $\begin{array}{c}\text { FNN-SVBL } \\
(\mathrm{FR})\end{array}$ & $\begin{array}{c}\text { SVFC-AK } \\
(\mathrm{FR})\end{array}$ & $\begin{array}{r}\text { RBF-SVM } \\
(\mathrm{SV})\end{array}$ & $\begin{array}{c}\text { SVFNN } \\
(\mathrm{FR})\end{array}$ & $\begin{array}{c}\text { SOTFN-SV } \\
(\mathrm{FR})\end{array}$ & $\begin{array}{c}\text { FNN } \\
(\mathrm{FR})\end{array}$ \\
\hline Iris & 3 & 3 & 16 & 9 & 4 & $n r^{*}$ \\
Vehicle & 9 & 9 & 302 & 125 & 221 & $n r$ \\
DNA & 3 & 3 & 967 & 334 & $n r$ & $n r$ \\
Satimage & 78 & 78 & 1611 & 889 & $n r$ & $n r$ \\
Glass & 7 & 7 & 113 & $n r$ & $n r$ & $n r$ \\
Wine & 3 & 3 & 56 & $n r$ & $n r$ & $n r$ \\
\hline
\end{tabular}

${ }^{*} n r:$ is not reported.

(SVFNN), self-organizing TS-type fuzzy network with support vector learning (SOTFN-SV) [32], support vector learning (SOTFN-SV) 64] and FNN classifier [72, 50]. The results of this comparison are displayed in Fig. 4. Overall, the method FNN-SVBL achieves the highest classification performance among all methods while having the smallest number of fuzzy rules along with SVFC-AK. For Iris dataset, FNN-SVBL achieves the same error rate (1.3\%) as SVFC-AK and they both have the lowest error rate compared to other methods. For DNA dataset, FNN-SVBL obtains an error rate of $4.1 \%$ which is slightly lower than the methods SVFC-AK (4.3\%) and RBF-SVM (4.6\%). For Glass dataset, FNN-SVBL achieves an error rate of $3.74 \%$. This is significantly lower than the error rate of SVFC-AK $(23.9 \%)$ and RBF-SVM (28.5\%), while using only 7 fuzzy rules comparing to 113 support vectors for RBF-SVM. For wine dataset, SVFC-AK has no classification error using only 3 rules. With the same number of rules FNN-SVBL achieves an error rate of $1.12 \%$. Nevertheless, RBF-SVM obtains an error rate of $0.6 \%$ using 56 support vectors. For the Vehicle dataset, whereas the error rate of SVFC-AK is the smallest one (10.3\%) by using only 9 rules, SOTFN-SV reports an error rate of $11.1 \%$ using 221 rules. For the Satimage dataset, while RBF-SVM obtains the uppermost classification performance $(8.7 \%)$, it has the largest number of support vectors (1611) among all methods. In contrast, FNNSVBL reports a slightly higher error rate $(8.9 \%)$ using only 78 fuzzy rules, which is much lower than both the number of rules of RBF-SVM and the number of support vectors of SVFNN.

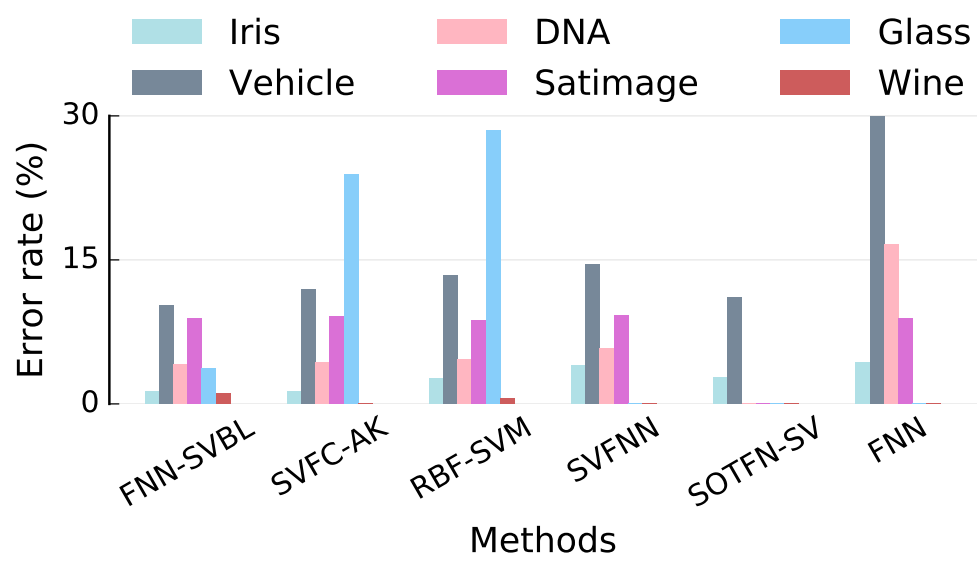

Figure 4: Comparison of classification error rate between FNN-SVBL and the related work methods. also in the number of its fuzzy rules, which is significantly lower than the related work. The results 
Table 3: Classification error rate obtained for different datasets by FNN-SVBL and the related work methods.

\begin{tabular}{cccrccc}
\hline Dataset & FNN-SVBL & SVFC-AK & RBF-SVM & SVFNN & SOTFN-SV & FNN \\
\hline Iris & 1.3 & 1.3 & 2.7 & 4 & 2.8 & 4.3 \\
Vehicle & 10.3 & 11.9 & 13.4 & 14.5 & 11.1 & 29.9 \\
DNA & 4.1 & 4.3 & 8.7 & 9.2 & $n r$ & 8.9 \\
Glass & 3.74 & 23.9 & 28.5 & $n r$ & $n r$ & $n r$ \\
Wine & 1.12 & 0.1 & 0.6 & $n r$ & $n r$ & $n r$ \\
\hline
\end{tabular}

${ }^{*} n r$ : is not reported.

reveal the effectiveness of the proposed adaptive kernel since by using fuzzy rules, FNN-SVBL can efficiently augment class separability in a kernel space.

For evaluation of FNN-SVBL performance in regression problem, we apply the method FNN-SVBL on the training set of nonlinear sinc function and Nonlinear dynamic system identification datasets according to the procedure explained earlier in this section. The resulting output is shown in Fig. 2b and $3 \mathrm{~b}$, respectively. The number of fuzzy rules is inspired from methods in [73] which represent the lowest RMSE for test sets in the related work. Table 4 and 5 report for both datasets, the numbers of neurons/fuzzy rules as well as the results of RMSE for training and test sets for FNN-SVBL and the related work. For the sake of clarity, the RMSE results are also displayed in Fig. 5a and 5a, For sinc function dataset, the number of rules used in FNN-SVBL is determined to 14 which is the lowest among all methods. For the dynamic system, the number of rules used in FNN-SVBL is determined to 9 which is comparable with methods such as [74] or space partitioning [73].

335 FNN-SVBL represents the highest regression performance for sinc function dataset with a training score of 0.0121 and a testing score equals to 0.0201. The same trend is observable for dynamic system dataset. The results of our method over training and test sets outperform the other methods.

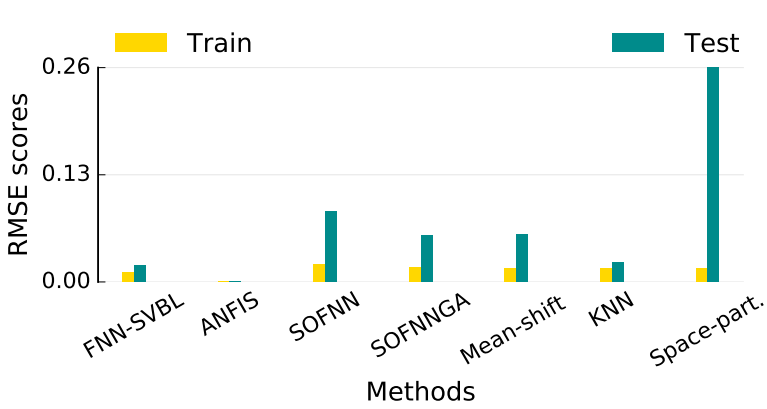

(a) Sinc function

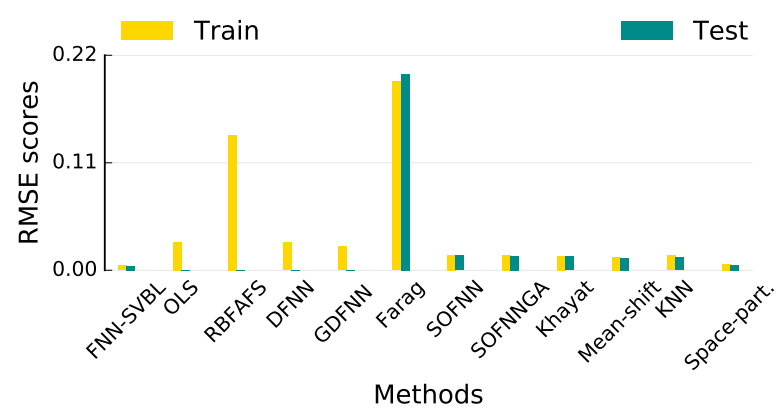

(b) Nonlinear dynamic system identification

Figure 5: Comparison of FNN-SVBL with the related work results.

We implement our method using the Python library. The computational experiments are carried out with a GTX 1070 Ti GPU based on Linux Fedora operating system.

\subsection{Fuzzy rules Sensitivity}

The parameters of FNN-SVBL is the number of fuzzy rules (clusters). Thus, the number of fuzzy rules is the only parameter to be investigated for sensitivity analysis. For this experimental study, we use Iris, Vehicle, DNA, Glass, and wine datasets for both classification and regression problems. To evaluate the sensitivity of FNN-SVBL to the number of fuzzy rules in a classification problem, we estimate the generalized accuracy using different number of rules in the range of $[2, \cdots, 16]$. Similarly, 
Table 4: Comparison of RMSE results for the training and testing sets for sinc function.

\begin{tabular}{cccc}
\hline Models & Neurons & Training RMSE & Testing RMSE \\
\hline FNN-SVBL & 14 & 0.0121 & 0.0201 \\
ANFIS [10] & 16 & $n r$ & $n r^{*}$ \\
SOFNN [25] & 14 & 0.0217 & 0.0860 \\
SOFNNGA [75] & 14 & 0.0173 & 0.0567 \\
Mean shift [73] & 18 & 0.0163 & 0.0571 \\
KNN [73] & 14 & 0.0168 & 0.0231 \\
Space partitioning [73] & 24 & 0.0161 & 0.2603 \\
\hline${ }^{*} n r:$ the result is not reported. & &
\end{tabular}

Table 5: Comparison of RMSE results for the training and testing sets for nonlinear dynamic system identification.

\begin{tabular}{cccr}
\hline Models & Neurons & Training RMSE & Testing RMSE \\
\hline FNN-SVBL & 9 & 0.0051 & 0.0042 \\
OLS & 65 & 0.0288 & $n r^{*}$ \\
RBFAFS & 35 & 0.1384 & $n r^{*}$ \\
DFNN [76] & 6 & 0.0283 & $n r^{*}$ \\
GDFNN [77] & 6 & 0.0241 & $n r^{*}$ \\
Farag & 75 & 0.193 & 0.201 \\
SOFNN [25] & 5 & 0.0157 & 0.0151 \\
SOFNNGA [75] & 4 & 0.0159 & 0.0146 \\
Khayat [74] & 4 & 0.0147 & 0.0141 \\
Mean shift [73] & 5 & 0.0137 & 0.0127 \\
KNN [73] & 4 & 0.0150 & 0.0131 \\
Space partitioning [73] & 9 & 0.0065 & 0.0055 \\
\hline${ }^{*} n r:$ the result is not reported. & &
\end{tabular}

to specify sensitivity of FNN-SVBL to the number of fuzzy rules in regression problem, we evaluate RMSE for the testing set using different number of rules in the range of $[6, \cdots, 18]$. The training sets of all datasets are scaled in the range of $[-1,1]$. To measure classification accuracy and RMSE for the datasets, which do not contain testing sets explicitly, we conduct a tenfold cross-validation on the whole training sets.

Figure 6a displays the classification accuracy of FNN-SVBL for different number of fuzzy rules. As it is shown, for Iris dataset the changes of fuzzy rules number dimly affect the accuracy. For Vehicle dataset, whereas the accuracy reaches its maximum value for 9 , it is gradually declined for the values 2,6 , and $[12, \cdots, 15]$. The sensitivity varies slightly for the values smaller than 5 as well as in the range between 10 and 13. For DNA dataset, the sensitivity is almost constant in the range of $[4, \cdots, 9]$. However, it achieves the best performance for the values 3 and 14. For Glass problem, there are more fluctuations than the other datasets though the overall sensitivity variations remain low. For Wine problem, the sensitivity to the fuzzy rules number is quasi-constant, except for the value 3 , which is representing the highest accuracy score among all values. Overall, FNN-SVBL shows a slight sensitivity to the variations of the number of fuzzy rules for the classification problem.

Figure 6a depicts the RMSE for the regression problem for different number of fuzzy rules. For sinc function dataset, while the RMSE score decreases for the value 14, it shows the highest score for the value 10. The global sensitivity is low excluding for the value 14. For dynamic system dataset, 


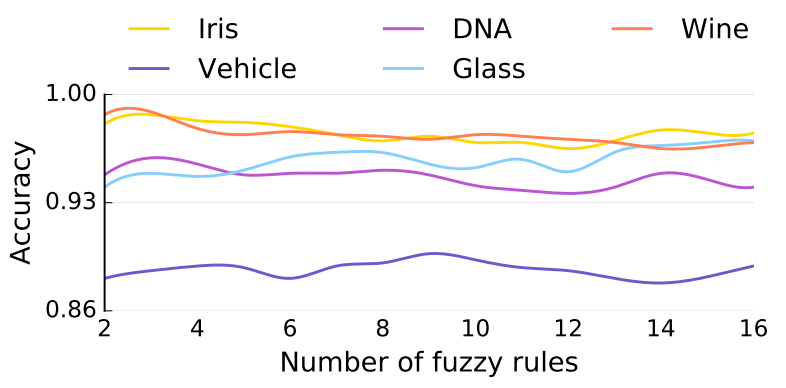

(a) Classification

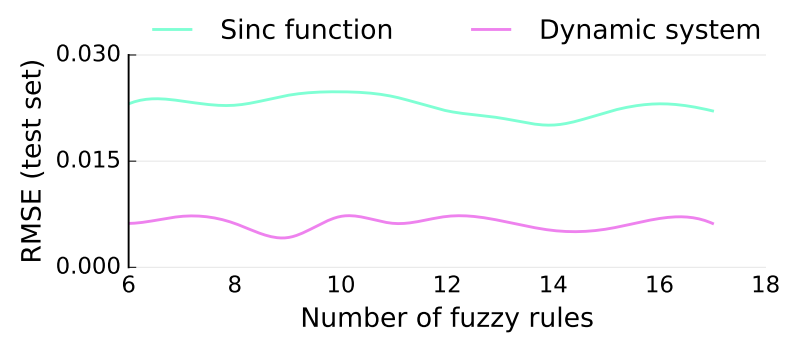

(b) Regression

Figure 6: The accuracy and RMSE of FNN-SVBL for different number of fuzzy rules.

the best performance belongs to the value 9 where the sensitivity tends to variate moderately. For the remaining values, the sensitivity stays steady.

In all datasets, the accuracy is ascending before and descending after the number of fuzzy rules chosen for our study. This complies with the choice of the number of fuzzy rules. Correspondingly, the effect of variation of fuzzy rules number on classification and regression performance of FNN-SVBL is predictable. Hence, using a cross-validation process the optimal values of fuzzy rules can be chosen by investigating a limited set of candidate values.

\subsection{Clustering Methods}

In this section, we address the problem of choosing the clustering method. To do so, we apply three most commonly used clustering GMM, FCM and HCA to obtain the FNN fuzzy rules (clusters). The number of clusters, as mentioned before, is inspired from [33. Then, the performance of FNN-SVBL is measured over the training sets of 10 benchmark datasets (for both classification and regression purposes) selected from UCI repository [34].

For classification purpose, the five benchmark datasets that are used for the study include student academics performance dataset, bank marketing, poker hand, human activity and breast cancer Wisconsin (diagnostic). The student academics performance dataset is composed of 22 attributes spanned across 300 instances. These multivariate attributes are chosen from different social, economic and academic characteristics such as students' gender, parents' degree, profession and wage, marital situation, social security type, students' major, to name a few. The classification goal is to predict the end semester students' performance (i.e. good, average or poor). The bank marketing dataset has 45,211 instances and 17 attributes. The input variables are related to social and economic situation of bank client data such as profession, marital and educational situation, housing, age and loan. Here, the aim of classification is to predict whether the client subscribes a bank product or not. The human activity recognition dataset contains information related to certain human activities (i.e. walking, walking-upstairs, walking-downstairs, sitting, standing, laying) that are collected using Smartphones. The dataset has 10,299 instances and 561 attributes providing the input data from sensor signals. The poker hand dataset consists of records for poker hands where each card is defined using two attributes of suit and rank for a total of 1,025,010 instances and 11 attributes. Here, the classification goal is to predict 10 poker hands class (ordinal) such as royal flush, full house, two pairs, straight flush. The breast cancer dataset comprises 32 attributes describing characteristics of 569 digitized images (instances) obtained from several patients' breast mass. Some of these attributes are radius, texture, compactness, fractal dimension or smoothness. The aim of classification is to diagnose whether the examined breast mass is malignant or benign.

For regression purpose, the five datasets that are used for the study include air quality [78, daily demand forecasting [79], energy efficiency [80, yacht hydrodynamics and individual household 
electric power consumption (IHEPC). The air quality dataset contains time-series information about

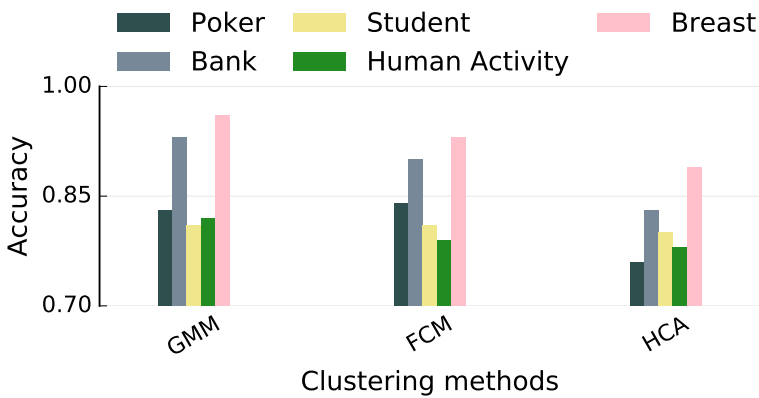

(a) Classification

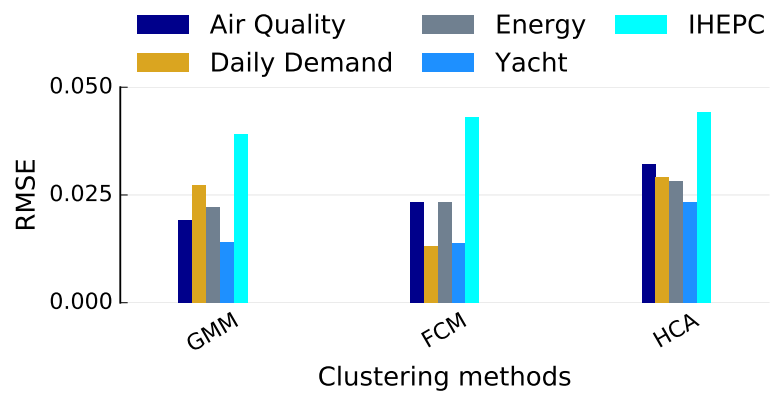

(b) Regression

Figure 7: The comparison of FNN-SVBL performance in classification and regression for 3 clustering methods (GMM, FCM and HCA).

As depicted in 7a, although GMM and FCM show similar scores in terms of accuracy for all datasets, the overall accuracy obtained by GMM is greater than FCM and HCA. This observation remains valid for regression scores. Generally, the method GMM reports the scores slightly lower than FCM and strictly lower than HCA (see 7b).

\section{Conclusion}

In this paper, we show that a TSK-type fuzzy neural network (TSK-FNN) can be equivalent to a support vector machine with an adaptive fuzzy kernel. It is also proved that this adaptive fuzzy kernel is generated by fuzzy rules used in the fuzzy neural network. In other words, we show that the weights in the latest layer of the TSK-FNN can be written based on the support vector machine parameters. Therefore, in order to obtain the weights in the latest layer of FNN, instead of using the gradient or pseudo-inverse method, one obtains the support vector machine parameters on the basis of which the weights of the latest layer of the TSK-FNN can be achieved. On the one hand, since FNN-SVBL is an SVM-based learning method, it takes advantage of SVM, which is the generalization ability (i.e., minimization of the test set error). This advantage is experimentally confirmed as reported in the paper. The proposed method achieves a higher performance compared to the state-of-the-art methods in terms of classification accuracy and regression error of the test sets. On the other hand, the proposed method outperforms the conventional SVM with standard kernels. In such kernels, choosing an appropriate kernel and learning kernel parameters are usually done empirically which are both cost-intensive and not optimal. While the kernel of the proposed method is based on data space since it is generated using fuzzy rules. As a result, it is expected that the results of the proposed method have a higher performance compared to an SVM with the standard kernel. The experimental outcomes 
of applying the proposed method for classification and regression and comparing their results with the conventional SVM with the standard kernels confirm this expectation. As reported in the paper, the proposed method demonstrates better results compared with the standard kernels in terms of the classification accuracy and regression error. Since the proposed method uses fuzzy rules to generate a kernel, and the kernel is, in fact, a similarity measure, the proposed method is not sensitive to the number of rules, and it is only sufficient that the number of rules is such that it can measure the similarity of each data with the support vectors. The insensitivity of the proposed method to the number of rules is also apparent from the experiments conducted for classification and regression. As shown in these results, there is no significant change for a different number of rules. In the future work, a conceivable way is to investigate the other fuzzy neural networks to know whether it is possible to find an adaptive kernel for a fuzzy neural network that can convert it to an SVM with a particular kernel.

\section{References}

[1] V. Vapnik, The nature of statistical learning theory, Springer Science \& Business Media, 2013.

[2] N. Cristianini, J. Shawe-Taylor, et al., An introduction to support vector machines and other kernel-based learning methods, Cambridge university press, 2000.

[3] O. Chapelle, V. Vapnik, O. Bousquet, S. Mukherjee, Choosing multiple parameters for support vector machines, Machine Learning 46 (1-3) (2002) 131-159.

[4] N. Cristianini, J. Shawe-Taylor, A. Elisseeff, J. S. Kandola, On kernel-target alignment, in: Advances in Neural Information Processing Systems, 2002, pp. 367-373.

[5] G. R. Lanckriet, N. Cristianini, P. Bartlett, L. E. Ghaoui, M. I. Jordan, Learning the kernel matrix with semidefinite programming, Journal of Machine Learning Research 5 (Jan) (2004) $27-72$.

[6] S. A. Ong CS, Williamson RC, Learning the kernel with hyperkernels, Journal of Machine Learning Research (2005) 1043-1071.

[7] A. Rakotomamonjy, F. R. Bach, S. Canu, Y. Grandvalet, Simplemkl, Journal of Machine Learning Research 9 (Nov) (2008) 2491-2521.

[8] B. Kosko, Fuzzy systems as universal approximators, IEEE Transactions on Computers 43 (11) (1994) 1329-1333.

[9] D. Nauck, R. Kruse, Function approximation by nefprox, in: Proc. Second European Workshop on Fuzzy Decision Analysis and Neural Networks for Management, Planning, and Optimization (EFDAN97), 1997, pp. 160-169.

[10] J.-S. Jang, Anfis: adaptive-network-based fuzzy inference system, IEEE Transactions on Systems, Man, and Cybernetics 23 (3) (1993) 665-685.

[11] R. J. Schilling, J. J. Carroll, A. F. Al-Ajlouni, Approximation of nonlinear systems with radial basis function neural networks, IEEE Transactions on Neural Networks 12 (1) (2001) 1-15.

[12] T. Takagi, M. Sugeno, Fuzzy identification of systems and its applications to modeling and control, IEEE Transactions on Systems, Man, and Cybernetics (1) (1985) 116-132.

[13] D. Nauck, R. Kruse, Neuro-fuzzy systems for function approximation, Fuzzy Sets and Systems 101 (2) (1999) 261-271. 
[14] M. M. Ebadzadeh, A. Salimi-Badr, Cfnn: correlated fuzzy neural network, Neurocomputing 148 (2015) 430-444.

[15] C.-T. Lin, C. G. Lee, C.-T. Lin, C. Lin, Neural fuzzy systems: a neuro-fuzzy synergism to intelligent systems, Vol. 205, Prentice hall PTR Upper Saddle River NJ, 1996.

[16] Y. Chai, L. Jia, Z. Zhang, Mamdani model based adaptive neural fuzzy inference system and its application, International Journal of Computational Intelligence 5 (1) (2009) 22-29.

[17] B. Gabrys, A. Bargiela, General fuzzy min-max neural network for clustering and classification, IEEE Transactions on Neural Networks 11 (3) (2000) 769-783.

[18] J. A. Dickerson, B. Kosko, Fuzzy function approximation with ellipsoidal rules, IEEE Transactions on Systems, Man, and Cybernetics, Part B: Cybernetics 26 (4) (1996) 542-560.

[19] A. Kroll, Identification of functional fuzzy models using multidimensional reference fuzzy sets, Fuzzy Sets and Systems 80 (2) (1996) 149-158.

[20] F. Klawonn, R. Kruse, Constructing a fuzzy controller from data, Fuzzy Sets and Systems 85 (2) (1997) 177-193.

[21] S.-K. Oh, W.-D. Kim, W. Pedrycz, B.-J. Park, Polynomial-based radial basis function neural networks (p-rbf nns) realized with the aid of particle swarm optimization, Fuzzy Sets and Systems 163 (1) (2011) 54-77.

[22] J. González, H. Rojas, J. Ortega, A. Prieto, A new clustering technique for function approximation, IEEE Transactions on Neural Networks 13 (1) (2002) 132-142.

[23] J.-S. Wang, C. G. Lee, Efficient neuro-fuzzy control systems for autonomous underwater vehicle control, in: Robotics and Automation, 2001. Proceedings 2001 ICRA. IEEE International Conference on, Vol. 3, IEEE, 2001, pp. 2986-2991.

[24] C.-C. Chuang, S.-F. Su, S.-S. Chen, Robust tsk fuzzy modeling for function approximation with outliers, IEEE Transactions on Fuzzy Systems 9 (6) (2001) 810-821.

[25] G. Leng, G. Prasad, T. M. McGinnity, An on-line algorithm for creating self-organizing fuzzy neural networks, Neural Networks 17 (10) (2004) 1477-1493.

[26] C.-W. Hsu, C.-J. Lin, A comparison of methods for multiclass support vector machines, IEEE Transactions on Neural Networks 13 (2) (2002) 415-425.

[27] M. Ester, H.-P. Kriegel, J. Sander, X. Xu, et al., A density-based algorithm for discovering clusters in large spatial databases with noise., in: Kdd, Vol. 96, 1996, pp. 226-231.

[28] Y. Chen, J. Z. Wang, Support vector learning for fuzzy rule-based classification systems, IEEE Transactions on Fuzzy Systems 11 (6) (2003) 716-728.

[29] J.-H. Chiang, P.-Y. Hao, Support vector learning mechanism for fuzzy rule-based modeling: a new approach, IEEE Transactions on Fuzzy systems 12 (1) (2004) 1-12.

[30] C.-F. Juang, S.-H. Chiu, S.-W. Chang, A self-organizing ts-type fuzzy network with support vector learning and its application to classification problems, IEEE Transactions on Fuzzy Systems 15 (5) (2007) 998-1008. 
[31] C.-T. Lin, C.-M. Yeh, C.-F. Hsu, et al., Fuzzy neural network classification design using support vector machine., in: ISCAS (5), 2004, pp. 724-727.

[32] C.-T. Lin, C.-M. Yeh, S.-F. Liang, J.-F. Chung, N. Kumar, Support-vector-based fuzzy neural network for pattern classification, IEEE Transactions on Fuzzy Systems 14 (1) (2006) 31-41.

[33] H. Ganji, S. Khadivi, M. M. Ebadzadeh, Support vector-based fuzzy classifier with adaptive kernel, Neural Computing and Applications (2017) 1-14.

515 [34] C. Blake, C. Merz, UCI Machine Learning Repository, http://archive.ics.uci.edu/ml/ datasets/, [Online; accessed 19-July-2018] ((1998)).

[35] M. D, S. DJ, T. CC, Machine learning, neural and statistical classification, ftp.stams.strath. ac.uk/pub/Statlog/pub//, [Online; accessed 19-July-2018] ((1994)).

[36] H.-C. Huang, Y.-Y. Chuang, C.-S. Chen, Multiple kernel fuzzy clustering, IEEE Transactions on Fuzzy Systems 20 (1) (2012) 120-134.

[37] Y. Kanzawa, Y. Endo, S. Miyamoto, On kernel fuzzy c-means for data with tolerance using explicit mapping for kernel data analysis, in: Fuzzy Systems (FUZZ), 2010 IEEE International Conference on, IEEE, 2010, pp. 1-6.

[38] J. Wang, J. Hua, J. Guo, Fuzzy maximum scatter discriminant analysis with kernel methods, in: Fuzzy Systems and Knowledge Discovery (FSKD), 2010 Seventh International Conference on, Vol. 2, IEEE, 2010, pp. 560-564.

[39] G. Heo, P. Gader, Robust kernel discriminant analysis using fuzzy memberships, Pattern Recognition 44 (3) (2011) 716-723.

[40] J. Guevara, Supervised machine learning with kernel embeddings of fuzzy sets and probability measures, Ph.D. thesis, IME USP (2016).

[41] J. Guevara, R. Hirata, S. Canu, Positive definite kernel functions on fuzzy sets, in: Fuzzy Systems (FUZZ-IEEE), 2014 IEEE International Conference on, IEEE, 2014, pp. 439-446.

[42] J. Guevara, R. Hirata, S. Canu, Cross product kernels for fuzzy set similarity, in: Fuzzy Systems (FUZZ-IEEE), 2017 IEEE International Conference on, IEEE, 2017, pp. 1-6.

[43] D. Zhang, M. Kamel, M. I. Elmasry, Fuzzy clustering neural network (fcnn): competitive learning and parallel architecture, Journal of Intelligent \& Fuzzy Systems 2 (4) (1994) 289-298.

[44] S. Wang, K. F. Chung, D. Zhaohong, H. Dewen, Robust fuzzy clustering neural network based on -insensitive loss function, Applied Soft Computing 7 (2) (2007) 577-584.

[45] H. Xiong, M. Swamy, M. O. Ahmad, Optimizing the kernel in the empirical feature space, IEEE Transactions on Neural Networks 16 (2) (2005) 460-474.

[46] S. Zhong, D. Chen, Q. Xu, T. Chen, Optimizing the gaussian kernel function with the formulated kernel target alignment criterion for two-class pattern classification, Pattern Recognition 46 (7) (2013) 2045-2054.

[47] P. Sollich, Bayesian methods for support vector machines: Evidence and predictive class probabilities, Machine Learning 46 (1-3) (2002) 21-52. 
[48] N. M. Khan, R. Ksantini, I. S. Ahmad, L. Guan, Sn-svm: a sparse nonparametric support vector machine classifier, Signal, Image and Video Processing 8 (8) (2014) 1625-1637.

[49] C. Cortes, M. Mohri, A. Rostamizadeh, Two-stage learning kernel algorithms., in: ICML, 2010, pp. 239-246.

[50] M. M. Lee, S. S. Keerthi, C. J. Ong, D. DeCoste, An efficient method for computing leave-one-out error in support vector machines with gaussian kernels, IEEE Transactions on Neural Networks 15 (3) (2004) 750-757.

[51] F. Aiolli, M. Donini, Easymkl: a scalable multiple kernel learning algorithm, Neurocomputing 169 (2015) 215-224.

[52] M. Kloft, U. Brefeld, S. Sonnenburg, A. Zien, Lp-norm multiple kernel learning, Journal of Machine Learning Research 12 (Mar) (2011) 953-997.

[53] A. Argyriou, C. A. Micchelli, M. Pontil, Learning convex combinations of continuously parameterized basic kernels, in: International Conference on Computational Learning Theory, Springer, 2005, pp. 338-352.

[54] P. Jawanpuria, J. S. Nath, G. Ramakrishnan, Generalized hierarchical kernel learning, The Journal of Machine Learning Research 16 (1) (2015) 617-652.

[55] C.-F. Lin, S.-D. Wang, Fuzzy support vector machines, IEEE Transactions on Neural Networks 13 (2) (2002) 464-471.

[56] R. Batuwita, V. Palade, Fsvm-cil: fuzzy support vector machines for class imbalance learning, IEEE Transactions on Fuzzy Systems 18 (3) (2010) 558-571.

[57] Y. Wang, S. Wang, K. K. Lai, A new fuzzy support vector machine to evaluate credit risk, IEEE Transactions on Fuzzy Systems 13 (6) (2005) 820-831.

[58] Q. Fan, Z. Wang, D. Li, D. Gao, H. Zha, Entropy-based fuzzy support vector machine for imbalanced datasets, Knowledge-Based Systems 115 (2017) 87-99.

[59] U. Ekong, H. Lam, B. Xiao, G. Ouyang, H. Liu, K. Y. Chan, S. H. Ling, Classification of epilepsy seizure phase using interval type-2 fuzzy support vector machines, Neurocomputing 199 (2016) $66-76$.

[60] E. Esme, B. Karlik, Fuzzy c-means based support vector machines classifier for perfume recognition, Applied Soft Computing 46 (2016) 452-458.

[61] D. Tsujinishi, S. Abe, Fuzzy least squares support vector machines for multiclass problems, Neural Networks 16 (5-6) (2003) 785-792.

[62] Z. Wu, H. Zhang, J. Liu, A fuzzy support vector machine algorithm for classification based on a novel pim fuzzy clustering method, Neurocomputing 125 (2014) 119-124.

[63] C.-F. Juang, G.-C. Chen, A ts fuzzy system learned through a support vector machine in principal component space for real-time object detection, IEEE Transactions on Industrial Electronics 59 (8) (2012) 3309-3320.

[64] C.-F. Juang, A tsk-type recurrent fuzzy network for dynamic systems processing by neural network and genetic algorithms, IEEE Transactions on Fuzzy Systems 10 (2) (2002) 155-170. 
[65] C.-F. Juang, C.-T. Lin, An online self-constructing neural fuzzy inference network and its applications, IEEE Transactions on Fuzzy Systems 6 (1) (1998) 12-32.

[66] J. Guevara, R. Hirata Jr, S. Canu, Kernel functions in takagi-sugeno-kang fuzzy system with nonsingleton fuzzy input, in: 2013 IEEE International Conference on, 2013, pp. 1-8.

[67] C. Fraley, A. E. Raftery, Model-based clustering, discriminant analysis, and density estimation, Journal of the American statistical Association 97 (458) (2002) 611-631.

[68] C. Fraley, A. E. Raftery, How many clusters? which clustering method? answers via model-based cluster analysis, The Computer Journal 41 (8) (1998) 578-588.

[69] N. Vlassis, A. Likas, A greedy em algorithm for gaussian mixture learning, Neural Processing Letters 15 (1) (2002) 77-87.

[70] G. Karypis, E.-H. Han, V. Kumar, Chameleon: Hierarchical clustering using dynamic modeling, Computer 32 (8) (1999) 68-75.

[71] S. Aeberhard, D. Coomans, O. de Vel, The classification performance of rda, Dept. of Computer Science and Dept. of Mathematics and Statistics, James Cook University of North Queensland, Tech. Rep (1992) 92-01.

[72] M. R. Berthold, J. Diamond, Constructive training of probabilistic neural networks, Neurocomputing 19 (1-3) (1998) 167-183.

[73] H. Malek, M. M. Ebadzadeh, M. Rahmati, Three new fuzzy neural networks learning algorithms based on clustering, training error and genetic algorithm, Applied Intelligence 37 (2) (2012) 280289.

[74] O. Khayat, M. M. Ebadzadeh, H. R. Shahdoosti, R. Rajaei, I. Khajehnasiri, A novel hybrid algorithm for creating self-organizing fuzzy neural networks, Neurocomputing 73 (1-3) (2009) $517-524$.

[75] G. Leng, T. M. McGinnity, G. Prasad, Design for self-organizing fuzzy neural networks based on genetic algorithms, IEEE Transactions on Fuzzy Systems 14 (6) (2006) 755-766.

[76] S. Wu, M. J. Er, Dynamic fuzzy neural networks-a novel approach to function approximation, IEEE Transactions on Systems, Man, and Cybernetics, Part B (Cybernetics) 30 (2) (2000) 358364.

[77] S. Wu, M. J. Er, Y. Gao, A fast approach for automatic generation of fuzzy rules by generalized dynamic fuzzy neural networks, IEEE Transactions on Fuzzy Systems 9 (4) (2001) 578-594.

[78] S. De Vito, E. Massera, M. Piga, L. Martinotto, G. Di Francia, On field calibration of an electronic nose for benzene estimation in an urban pollution monitoring scenario, Sensors and Actuators B: Chemical 129 (2) (2008) 750-757.

[79] R. P. Ferreira, A. Martiniano, A. Ferreira, A. Ferreira, R. J. Sassi, Study on daily demand forecasting orders using artificial neural network, IEEE Latin America Transactions 14 (3) (2016) $1519-1525$.

[80] A. Tsanas, A. Xifara, Accurate quantitative estimation of energy performance of residential buildings using statistical machine learning tools, Energy and Buildings 49 (2012) 560-567. 